\title{
Effect on Suppression of Biofilm Growth using Microstructures Inspired by Living Organism
}

\author{
Mariko Miyazaki $^{{ }^{*}}$ and Akihiro Miyauchi ${ }^{2}$ \\ ${ }^{1}$ Research and Development Group, Hitachi, Ltd., \\ 7-1-1 Omika-cho, Hitachi, Ibaraki 319-1292, Japan \\ ${ }^{2}$ Institute of Biomaterials and Bioengineering, Tokyo Medical and Dental University, \\ 2-3-10 Kanda-Surugadai, Chiyodaku, Tokyo 101-0062, Japan \\ *mariko.miyazaki.jm@hitachi.com
}

\begin{abstract}
A technique for antibacterial functions is always required in such as medical treatment or food manufacturing. Sharkskin, wings of cicadas and dragonflies have antibacterial functions on their surfaces. It is attractive for human technologies to mimic their microstructural surfaces. In this study, we cultured biofilms on micro-pillar surfaces which was inspired by living organism, and evaluated the antibacterial effect. Growth of biofilms depended on the pitch and diameter of pillar arrays. Formations of the biofilm were suppressed when the pitch and diameter of the pillars were comparable to the size of the bacteria. This suppression seems to be caused by the instability of bacteria sticking on the pillar-array scaffold.

Keywords: Biomimetic riblets, Sharkskin, 3D digitizing, Antibacterial effect, Biofilm, Fluid control
\end{abstract}

\section{Introduction}

There has been remarkable progress in biomimetics, the emulation of nature's time-tested patterns, functions, and strategies for the purpose of discovering sustainable solutions to practical problems in recent years [1]. For example, the adhesive function of geckos' feet has been studied [2]. Geckos are known to crawl up walls, hang from ceilings, and firmly grasp slippery glass-like surfaces using their adsorptive toes. The flagella on the soles of geckos' feet are dense, and the feet adhere by van der Waals forces working between the flagella and the surface. In addition, an excellent water-repellent surface was developed that was inspired by the fine, rough surface texture of lotus leaves [3].

With respect to antibacterial effects, two surface structures of living matter are known as having antibacterial functions.

One is the nano-structural pillar surface on the wings of cicadas and dragonflies [4-6]. Ivanova et al. found that the surfaces of both black silicon and wings of Diplacodes bipunctata have a strong antibacterial effect that were confirmed by Gramnegative (Pseudomonas aeruginosa) and Gram- positive (Staphylococcus aureus, Bacillus subtilis) bacteria [4]. Both surfaces have a hierarchical surface-structure through the formation of clusters of adjacent nano-protrusions. These nanostructures are significantly smaller than the size of bacteria, and reported that antibacterial effect is caused by physical breaking of the cell membranes of the bacteria.

Another is the denticles of shark skin. Sharkskin is covered with numerous small tooth-like elements termed "dermal denticles" ("denticles" hereafter) [7, 8]. The microstructure of denticles (i.e., the minute projections) are reported in view of controlling a turbulence flow and reducing drag experimentally and computationally [9-24]. We reported that there is a special flow, vertical flow near the denticles, and this flow beark the bacteria film and suppress the growth of biofilm $[25,26]$. Also an antibacterial plate (Sharklet ${ }^{\mathrm{TM}}$ ) made of silicone elastomer has been known to provide an antibacterial effect in various pathogenic microorganisms (Staphylococcus aureus, methicillin-resistant Staphylococcus aureus, Pseudomonas aeruginosa, Escherichia coli, and vancomycin-resistant enterococci) [27, 28]. 
Considering the feature size of functional surfaces of living matters, the micron-scale such as the flagella of geckos' feet or denticles of shark skin is cell-size because these structures are generated by cell. On the other side, the surface structures of lotus leave or wings of cicadas and dragonflies are submicron scale and formed by self-organization of proteins or organics.

We focused self-organized structures, namely concave-convex surface, in this research, and analyze the antibacterial effect using artificially designed pillar arrays as a self-organized surface.

\section{Experiment}

2.1. Fabrication of pillar arrays

Pillar arrays for a bacterial culture were prepared using nanoimprinting [32]. Figure 1 shows the fabrication process of nanoimprint. A mold has fine hole patterns on its surface. The mold is pressed to a resin film at the temperature around a glasstransition of resin film. Then the mold is de-molded from the film, and the designed pillar array were formed on the film surface.

Figure 2 shows a schematic diagram of a mold on which the micro-patterned hole structures were fabricated. A conventional optical lithography and silicon etching were used to fabricate the mold. Figure 2 (a) shows a perspective view of the mold. The mold size is $28 \times 28 \times 2 \mathrm{~mm}$, and half of the area was the fabrication area. Figure 2 (b) shows a top view of the mold. $1 \mu \mathrm{m}$-diameter holes (about the same size as bacteria) and $5 \mu \mathrm{m}$-depth were fabricated. The mold was divided into eight regions of $4 \mathrm{~mm} \times 4 \mathrm{~mm}$, and each differed in the pitch (a distance between the centers of the nearest holes). The pitch size were 1.5, 1.6, 1.7, 1.8, 1.9, 2.0, 2.2, and $2.4 \mu \mathrm{m}$. Using this mold, pillar arrays on resin films were obtained by nanoimprint.

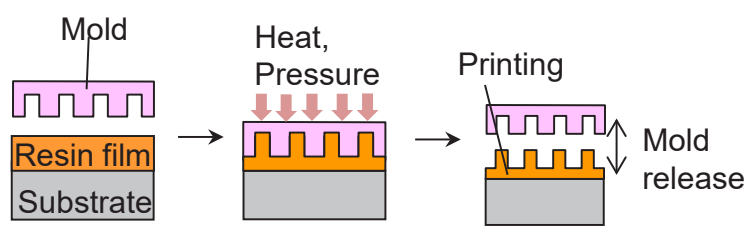

Fig. 1. Method for fabricating resin sheet by nanoimprinting.
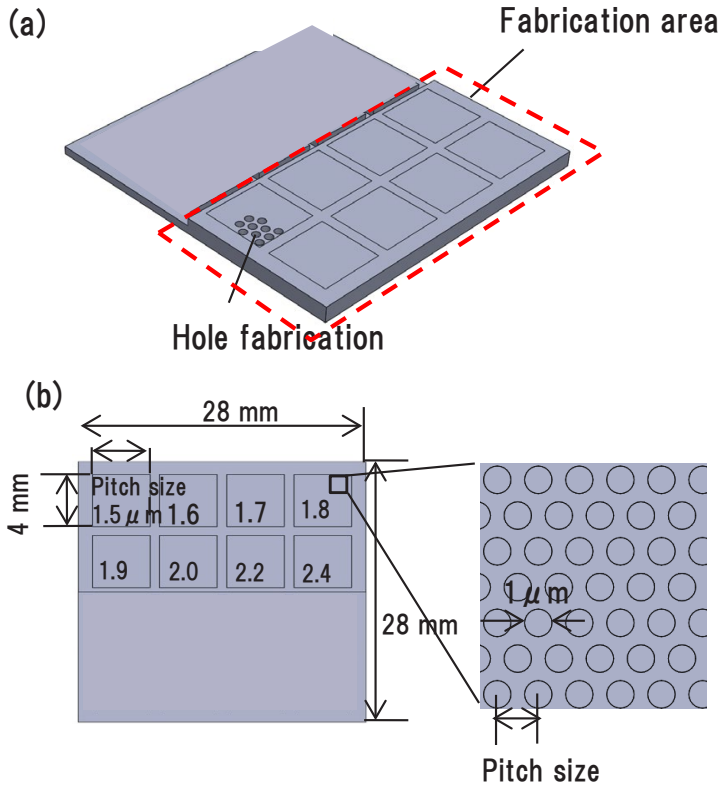

Fig. 2. Schematic diagram of mold for pillar arrays. (a) Perspective view. (b) Top views.

\subsection{Method of bacterial culture}

A culture device was designed for the pillar arrays. Figure 3 shows a CAD image and appearance of the designed culture device. A resin container which fix the pillar-array chips was placed in a glass petri dish with an outer diameter of $90 \mathrm{~mm}$ and a height of 45 $\mathrm{mm}$. The container has four places to fix the pillararray chip. There was a glass-lid.

The bacterial culture conditions are shown in Table 1. Staphylococcus aureus subsp. aureus NBRC 15035 was used as test bacteria. Staphylococcus aureus is spherical and has a diameter of about $0.9 \mu \mathrm{m}$. The test bacteria was cultured in a trypticase soy (SCD) broth medium, and the number of cultured test bacteria was $10^{7}$ $10^{8} / \mathrm{mL}$ The medium was exchanged once a day. The temperature of an incubator was kept at $37^{\circ} \mathrm{C}$, and the cultured bacteria solution was obtained on the fifth day of the culture.

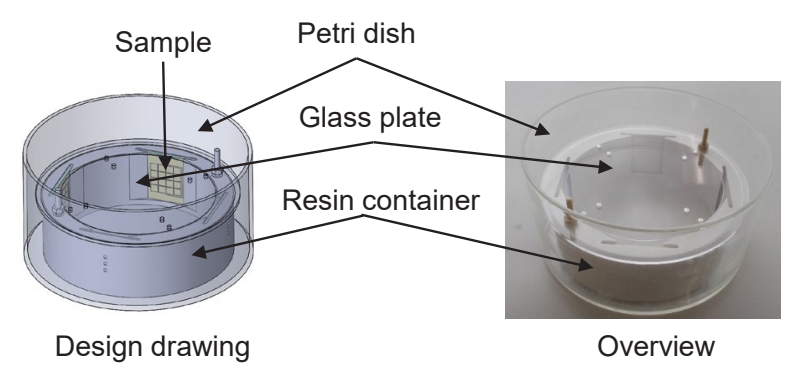

Fig. 3. CAD image and appearance of the culture device. 


\subsection{Quantification method of bacteria coverage}

A degree of biofilm-growth was evaluated by an optical image. After 5-day culture, the pillar array chips were removed from the culture device, and then Gram stain were treated [33]. The chips were immersed in crystal violet solutions for one minute and then rinsed with purified water.

The color-images of dyed chips were taken by an optical microscope. The degree of color indicates a thickness of biofilm, namely the image appears darker as the biofilm becomes thicker. The coverage ratio of biofilm was calculated from a binaryprocessed image of the chip. Each pixel of the taken image was binarized to white (not existing bacteria) and black (existing bacteria), and the covering ratio was obtained using the equation as follow.

$$
\begin{aligned}
& \text { covering ratio of bacteria } \\
& =\frac{\text { Area of black area }}{\text { Total area of image }} \times 100(\%)
\end{aligned}
$$

Table 1. Bacterial culture conditions

\begin{tabular}{|l|l|}
\hline $\begin{array}{l}\text { Bacteria } \\
\text { type }\end{array}$ & $\begin{array}{l}\text { Staphylococcus aureus subsp. } \\
\text { aureus NBRC } 15035\end{array}$ \\
\hline $\begin{array}{l}\text { Preparation } \\
\text { of bacteria } \\
\text { solution }\end{array}$ & $\begin{array}{l}\text { The bacteria are cultured in SCD } \\
\text { broth medium at } 37^{\circ} \mathrm{C} \text { for } 24 \text { hours, } \\
\text { and then suspended in SCD broth } \\
\text { medium to prepare test bacteria in } \\
\text { which the number of bacteria is } 10^{7} \\
\text { to } 10^{8} / \mathrm{mL} .\end{array}$ \\
\hline $\begin{array}{l}\text { Sterilization } \\
\text { process }\end{array}$ & $\begin{array}{l}\text { Sterilize test samples culturing } \\
\text { equipment under high pressure } \\
\text { steam sterilization (121 }{ }^{\circ} \mathrm{C}, 15 \\
\text { minutes) }\end{array}$ \\
\hline Biofilm & $\begin{array}{l}\text { Prepared bacteria solution (300 - } \\
\text { formation } \mathrm{mL} \text { ) was added to the culture } \\
\text { device. The solution was stirred for } \\
1 \text { minute using a magnetic stirrer. } \\
\text { The cultured device with pillar } \\
\text { arrays were rinsed by a purified } \\
\text { water three times, then add fresh } \\
\text { SOD broth medium (300 - 350 } \mathrm{mL} \text { ) } \\
\text { and cultured at } 37{ }^{\circ} \mathrm{C} \text {. This process } \\
\text { repeated once a day. }\end{array}$ \\
\hline
\end{tabular}

\section{Result and discussion}

Figure 4 (a) and (b) shows the SEM image after Staphylococcus aureus culture, and the pitch is 1.5 $\mu \mathrm{m}$ and $2.2 \mu \mathrm{m}$, respectively. Few bacteria (Staphylococcus aureus) were detected in $1.5 \mu \mathrm{m}$ pitch size. However, there were many bacteria in the gaps of the pillar structures observed like white round shapes in case of $2.2 \mu \mathrm{m}$ pitch array. Same distributions of bacteria were observed in the case of $2.0 \mu \mathrm{m}$ pitch array. When the pitch size was large than $2.0 \mu \mathrm{m}$, bacteria seems to exist between the pillars and form a biofilm easily.

Figure 5 shows the optical microscopic images (200x) of pillar arrays after 5-day bacterial culture and staining with crystal violet. The pillar structures cannot be observed in this low magnitude, and an average situation can be evaluated in wide area. The optical image becomes darker as increase of pitch size, namely the thickness of biofilm becomes thicker as increase of pitch size of pillar array. Figure 6 shows the relationship between the pitch size and the brightness of each array. The vertical axis is normalized by the value at the pitch size of $1.5 \mu \mathrm{m}$. The brightness is maximum when the pitch is lower than $1.7 \mu \mathrm{m}$. However, the brightness decreases when the pitch becomes less than $1.9 \mu \mathrm{m}$. This shows the growth situation of biofilm depends on the pitch of pillar array, and there is a critical pitch size around $1.7-1.9 \mu \mathrm{m}$. We add the comment that when observe the edge of pillar array region, plenty of stained areas were observed outer the pillar array region, namely a plenty of biofilm was grown on the flat area.

Next, we examined to calculate the coverage ratio of biofilm using equation (1). Figure 7 shows the relationship between the pitch size and the coverage ratio. The coverage ratio on the vertical axis was normalized by the value at the pitch size of $1.5 \mu \mathrm{m}$. This graph also shows that there is the critical pillar pitch around $1.7-1.9 \mu \mathrm{m}$.

As mentioned above, there is the critical pillar pitch around $1.7-1.9 \mu \mathrm{m}$ for growth of Staphylococcus aureus. Here considering the size of Staphylococcus aureus, namely around $0.9 \mu \mathrm{m}$, the suppression model of growth is shown in figure 8 . In general, biofilms are grown step by step. First a pioneer bacteria stick on a carbonated substrate, and then a next bacteria stick onto the first pioneer bacteria. Therefore, the important process of biofilm is the first coverage of pioneer bacteria onto the substrate. In our experiment, the first sticking of bacteria is restricted in case of a narrow spacing of pillar. Therefore, the continuous sticking of bacteria 
is restricted and a conventional growth process of biofilm seems to be inhibited.

\section{Conclusion}

Surface structures in micron-scale in nature is formed by self-organization phenomena. The artificially designed micron-structures were designed and pillar array chips were fabricated by nanoimprint. Staphylococcus aureus was cultured using the original culture device. The growth of Staphylococcus aureus was restricted when the pillar pitch is less than $1.7-1.9 \mu \mathrm{m}$, and the biofilm did not grow in these narrow pitch array chips. The restriction of inserting Staphylococcus aureus to base area of substrate seem to cause the inhabitation of biofilm growth process.
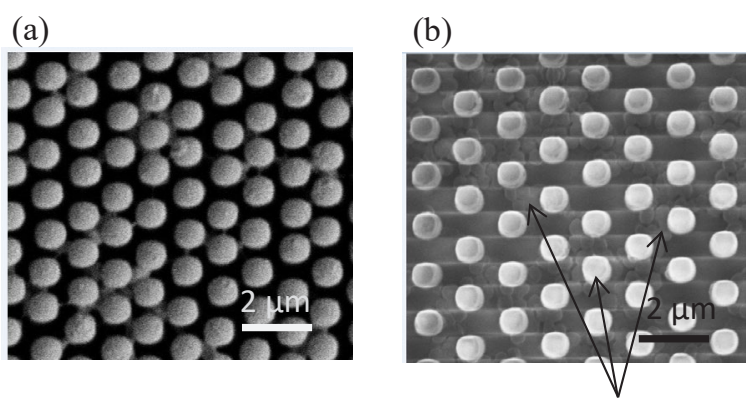

Staphylococcus aureus

Fig. 4. SEM images of pillar arrays after 5-day bacterial culture. Pitch size is (a) $2.2 \mu \mathrm{m}$, (b) $1.5 \mu \mathrm{m}$. (a)

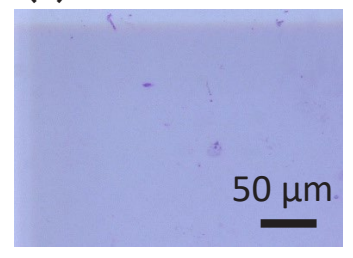

(c)

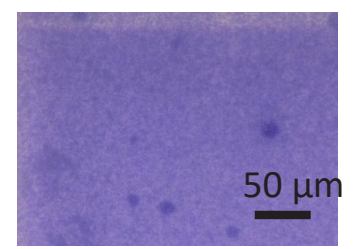

(b)

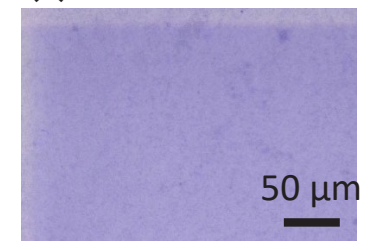

(d)

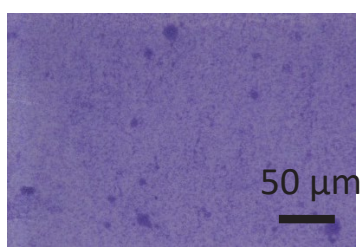

Fig. 5. Microscope images of pillar arrays after 5-day bacterial culture (200x). The chips were stained by crystal violet. The pitch is (a) $1.5 \mu \mathrm{m}$, (b) $1.7 \mu \mathrm{m}$, (c) 1.8 $\mu \mathrm{m}$, (d) $2.2 \mu \mathrm{m}$.

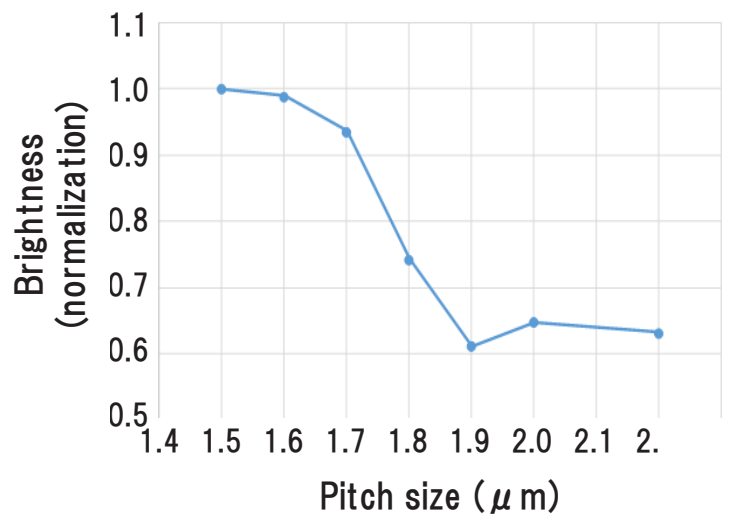

Fig.6. Relationship between pitch size and brightness of optical microscope images of cultured chips.

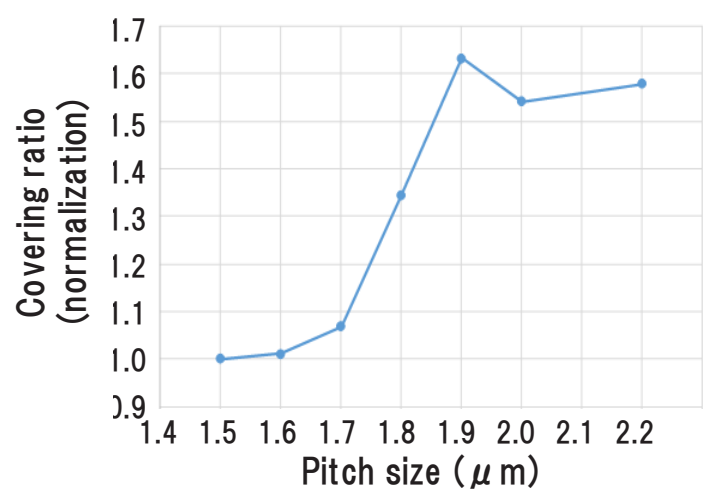

Fig.7. Caluclated coverage ratio of biofilm on cultured chips. (a)

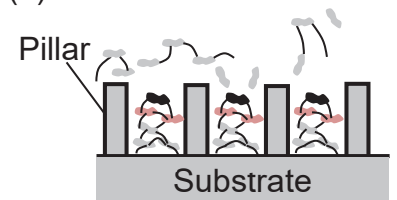

(b)

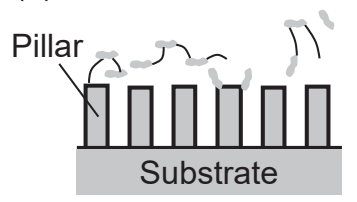

Fig.8. Model of biofilm growth. (a) In case of the pitch size is enough for inserting the bacteria, the bacteria stick onto the base substrate, then biofilm grows step by step by continuous sticking of bacteria. (b) In case of pitch size is narrower than the size of bacteria, the first bacteria is hard to stick onto the pillar-tops and hard to form the biofilm.

\section{Conclusion}

In this study, we focused on the antibacterial effect of surfaces inspired by living organism and evaluated the effect of suppressing biofilm growth on the scaffold with fabricated microstructural designs. Micro-patterned pillar structures were fabricated on a resin sheet by nanoimprint, on which Staphylococcus aureus was cultured for five days. The amount of attached bacteria was evaluated by calculating the brightness of the optical microscopic 
images of cultured samples stained with crystal violet. The micro-patterned pillar structures suppress the biofilm growth and decrease the bacteria covering when the pitch size of the pillars and the size of the bacteria are comparable. The antibacterial mechanism was proposed in which the concavo-convex structure of the surface makes unstable for bacteria sticking and the formation of the biofilm was suppressed when the pitch size of the pillars and the size of the bacteria were comparable.

\section{References}

1. H. Liu, T. Nakata, G. Li, and D. Kolomenskiy, Acta Mechanica Sinica, 33 (2017) 663.

2. K. Autumn, A. L. Yiching, S. T. Hsieh, W. Zesch, W. P. Chan, and T. W. Kenny, Nature, 405 (2000) 681.

3. E. P. Chan, C. Greiner, E. Arzt, and A. J. Crosby, MRS Bulletin, 32 (2007) 496.

4. E. Ivanova, J. Hasen, H. Webb, G. Gervinskas, S. Juodkazis, V. Truong, A. Wu, R. Lamb, V. Baulin, G. Watson, J. Watson, D. Mainwaring, and R. Crawford, Nature Communications, 4 (2013) 2838.

5. J. Hasen, H. Webb, V. Truong, S. Pogodin, V. Baulin, G. Watson, J. Watson, R. Crawford, and E. Ivanova, Appl. Microbiol. Biotechnol., 97 (2013) 9257.

6. K. Nakade, T. Sagawa, H. Kojima, T. Shimizu, and T. Ito, Material Today: Proceedings 7 (2019) 497.

7. L. Wen, J. C. Weaver, and G. V. Lauder, J. Exp. Biol., 217 (2014) 1656.

8. N. E. Kemp, "Integumentary System and Teeth in Sharks, Skates, and Rays: The Biology of Elasmobranch Fishes" (ed. W. C. Hamlet), Johns Hopkins University Press, 43 (1999).

9. W. Meyer and U. Seegers, J. Fish Biol., 80 (2012) 1940.

10. J. I. Castro, The Sharks of North America, Oxford University Press, (2011).

11. W. E. Reif and A. Dinkelacker, Neues Jahrb. Geol. Palaontol. Abh., 164 (1982) 184.

12. W. E. Reif, Squamation and Ecology of Sharks, Frankfurt am Main: Senckenbergische Naturforschende Gesellschaft, (1985).

13. D. W. Bechert, M. Bruse, W. Hage, van der Hoeven J, and G. Hoppe, J. Fluid Mech., 338 (1997) 59.

14. D. W. Bechert, M. Bruse, and W. Hage, Exp. Fluids, 28 (2000) 403.

15. A.W. Lang, P. Motta, R. Hueter, and M. Habegger, J. Mar. Technol. Soc., 45 (2011) 208.
16. M. J. Walsh, AIAA J., 72 (1980) 168.

17. M. J. Walsh, AIAA J., 21 (1983) 485.

18. P. Vukoslavcevic, J. M. Wallace, and J. I. Balint, AIAA J., 30 (1992) 1119.

19. H. Choi, P. Moin, and J. Kim J, J. Fluid Mech., 255 (1993) 503.

20. S. R. Park, AIAA J., 32 (1994) 31.

21. S. J. Lee and S. H. Lee, Exp. Fluids, 30 (2001) 153.

22. B. Dean and B. Bhushan, Phil. Trans. R. Soc., A368 (2010) 4775.

23. C. C. Buttner and U. Shulz, Smart Matter. Struct., 20 (2011) 094016.

24. Y. Luo, Y. Liu, D. Zhang, and EYK NG, $J$. Mech. Med. Biol., 14 (2014) 1450029.

25. M. Miyazaki, Y. Hirai, H. Moriya, M. Shimomura, A. Miyauchi, and H. Liu, J. Bionic Engineering, 15 (2018) 999.

26. M. Miyazaki and A. Miyauchi, J. Photopolymer Science and Technology, 32 (2019) 295.

27. K. K. Chung, J. F. Schumacher, E. M. Sampson, R. A. Burne, P. J. Antonelli, and A. B. Brennan, Biointerphases, 2 (2007) 89.

28. S. T. Reddy, K. K. Chung, C. J. McDaniel, R. O. Darouiche, J. Landman, and A. B. Brennan, $J$. Endourol., 25 (2011) 1547.

29. D. C. Mayette, WaterReview, Water Quality Research Council, Lisle Il (1992) 1.

30. S. B. Borenstein, "Microbiologically Influenced Corrosion Handbook", Industrial Press Inc., New York (1994).

31. A. Coghlan, New Scientist, 15 (1996) 32.

32. A. Miyauchi, "Nanoimprinting and its applications", Jenny Stanford Publishing (2019).

33. M. J. Wilhelm, J. B. Sheffield, M. S. Gh., Y. Wu, C. Spahr, G. Gonella, B. Xu, and H. L. Dai, ACS Chemical Biology, 10 (2015) 1711. 